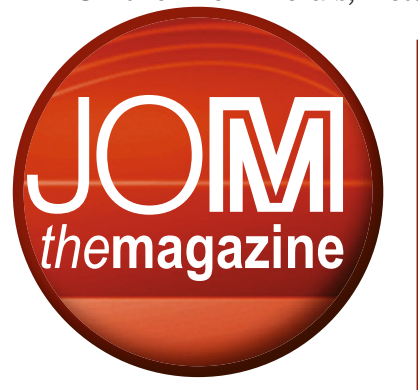

\title{
New Extractive Metallurgy Meeting Series; TMS Annual Business Meeting; Congratulations to ELA Participants
}

\section{new and noteworthy at TMS}

Turn to this regular JOM feature for information on new TMS initiatives, updates and overviews of TMS activities, and news from the field that impacts TMS and its members. To submit news items for consideration, contact Lynne Robinson, JOM Magazine Editor, at Irobinson@ @ms.org.

\section{Extraction 2018 Confirms Dates and Shares Initial Programming Plans}

A new meeting series focused on both the technical and business needs

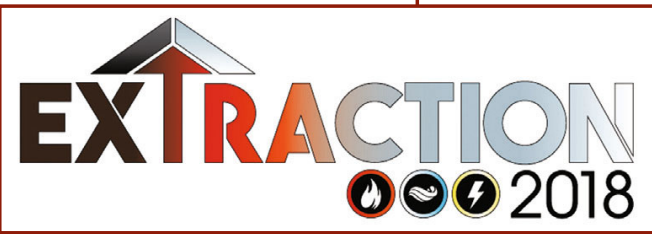

of the global extractive metallurgy community launches in 2018. Extraction 2018 will take place August 26-29, in Ottawa, Canada.It is organized by the Metallurgy and Materials Society (MetSoc) of the Canadian Institute of Mining, Metallurgy and Petroleum (CIM); the Society for Mining, Metallurgy \& Exploration (SME); and The Minerals, Metals \& Materials Society (TMS).

For information and to sign up for e-mail updates, visit extractionmeeting.org.

Extraction 2018 will host several important recurring symposia in the field, while also offering new programming tracks, opportunities to explore marketplace and business issues, a slate of social and networking events, and an industrial exhibition. At press time, the following technical symposia were confirmed:

- Hydrometallurgy 2018: A recurrent symposium organized by MetSoc, SME and TMS and held every five years. Hydrometallurgy 2018 provides a forum for the dissemination of research related to hydrometallurgical extraction and purification.

Organizing Committee Includes: Michael Free, University of Utah, USA, Chair

- Ritcey Symposium: A symposium honoring Gordon M. Ritcey, organized by MetSoc.
Organizing Committee Includes: Jeff Adams, Hatch, Canada, Chair; Ron Molnar, MetNetH2O Inc., Canada

- Advances in Sulfide Smelting: A recurrent symposium of the TMS Extraction \& Processing Division that encompasses advancements in all aspects of smelting technology, including basic principles, technology, and current operating practice.

Organizing Committee Includes: Gerardo Alvear, Aurubis, Germany, Chair; Mark Schlesinger, Missouri University of Science and Technology, USA

- Sulfide Flotation: A new symposium under development by SME.

Organizing Committee Includes: Tarun Bhambhani, Solvay, USA; Ronel Kappes, Newmont USA Ltd.

- Critical Materials Production: A new symposium under development by MetSoc.

Organizing Committee Includes: Niels Verbaan, SGS Minerals Services, Canada

- Extractive Metallurgy Markets and Economies: A new symposium under development by MetSoc, SME, and TMS. The goal of this program is to enlighten metallurgical researchers on the main economic drivers in the markets, to inform operating companies about world commodity trends in the next five years, and to make financial 
people aware of technology and environmental issues.

Organizing Committee Includes:Boyd

Davis, Kingston Process Metallurgy

Inc., Canada

The conference co-chairs are: Boyd Davis, Kingston Process Metallurgy Inc., Canada (MetSoc); Michael Moats,
Missouri University of Science and Technology, USA (SME); and Shijie Wang, Rio Tinto Kennecott Utah Copper Corporation., USA (TMS).

A call for abstracts will be issued in the coming months. Check back regularly at extractionmeeting.org for news and updates on the meeting.

\section{Deadline Approaching for NASA's 3D-Printed Habitat Challenge}

Registration for the Structural Member Competition component of NASA's 3D-Printed Habitat Challenge closes on January 31, 2017.

Teams will compete for a prize of $\$ 1.1$ million by developing a 3D printed habitat for use in deep space exploration. The structural components must be created using either a combination of indigenous and mission recycled materials or indigenous materials alone.

The Structural Member Competition is Phase 2 of three phases in a challenge aimed at advancing the technology of additive manufacturing and ultimately creating sustainable housing solutions for future implementation, both on and offworld.

Tracie J. Prater, a TMS member, joined NASA's 3D-Printed Habitat Challenge organizing team through her involvement with the in-space manufacturing project and additive manufacturing efforts at NASA Marshall. "Just in the past decade, we've seen additive manufacturing transform multiple industries, from aerospace to automotive, and construction is another sector where it's poised to make a unique impact." Prater said. "3D printing can enhance safety and improve productivity through remote and autonomous fabrication of structures, contribute to infrastructure improvements, and enable flexibility in materials and design not possible with traditional construction techniques."

Participating in the 3D-Printed Habitat Challenge is more than an opportunity to compete for a title, commented Prater, noting that involvement in the competition "represents an incredible opportunity to be part of the next revolution in manufacturing and construction."

For further information on the competition or to register a team, visit bradley.edu/sites/challenge.

\section{TMS Members Grow as Leaders at ELA}

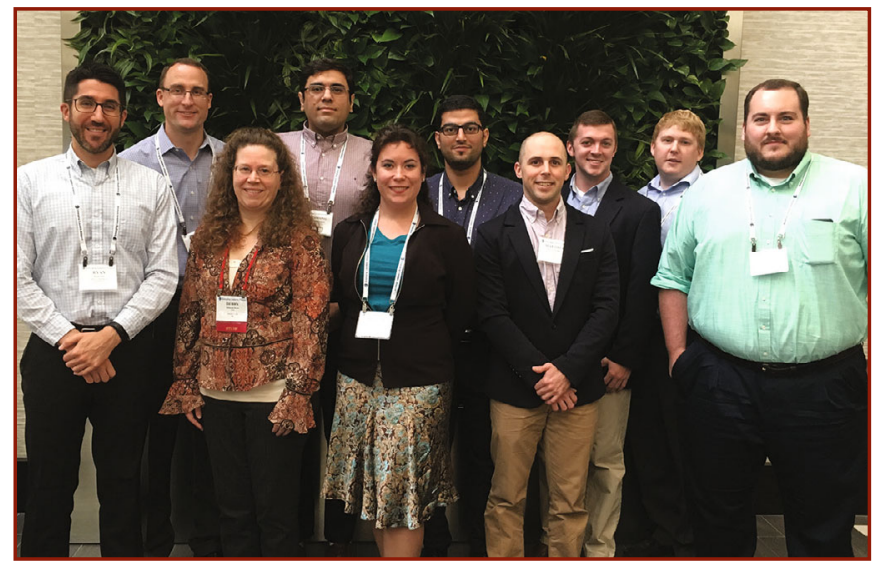

The TMS Foundation supported the participation of seven promising TMS members in the Emerging Leaders Alliance (ELA) leadership conference, held November 9-12, 2016, in Falls Church, Virginia. Those receiving the
TMS Foundation grants were: Patrick Bowen, DeringerNey; Keegan Hammond, Real Alloy; Matthew Humbert, Boston Electrometallurgical Corp.; Ryan Paul, GrafTech International; Behrang Poorganji, Eaton Corporation; Michael Powell, Southwire Company; and Douglas Spearot, University of Florida. A multi-society initiative, the ELA provides interdisciplinary leadership training to prepare engineers with potential for upper-level positions for the challenges that they will face in advancing their organizations within complex business and social environments. For information on how to apply to the 2017 program, contact Deborah Hixon, TMS Awards \& Recognition Specialist, at hixon@tms.org.

Pictured at the 2016 ELA conference are (front, left to right): Paul, Hixon, Hammond, Humbert, and Bowen. (Back, left to right): Spearot, Poorganji, Mohsen Mohammadijoo, University of Alberta (representing the American Institute of Mining, Metallurgical, and Petroleum Engineers (AIME)), Powell, and Chad Hornbuckle, Army Research Laboratory (representing AIME). 


\section{TMS Welcomes New Members}

Please join TMS in congratulating the following new members, approved by the TMS Board of Directors at its October 2016 meeting:

Abolghasem, Sepideh; University De Los Andes, Colombia

Abraham, Mark A.; Calumite Company, United States

Agudo Jácome, Leonardo; Bundesanstalt für Materialforschung und Prüfung, Germany

Almer, Jonathan; Argonne National Laboratories, United States

Bachmaier, Andrea; Erich Schmid Institute, Germany

Baguley, Roy; Metal Engineers International Inc., Canada

Bain, Andrew; United States

Balaji, A.K.; University of Utah, United States

Balci, Ozgur; United States

Barber, Lee P.; Wyman-Gordon Company, United States

Benayas, Antonio; National Institute of Research Science-Energy, Materials and Telecommunications, Canada

Bentria, El Tayeb; Algeria

Booth-Morrison, Christopher; Siemens, Canada

Chen, Zhe; University of California Santa Barbara, United States

Cheng, Ka Wing; Master Dynamic Limited, Hong Kong

Chesnes, Richard P.; TCR Technologies Ltd, United States

Choudhury, Abhik Narayan; Indian Institute of Science, India

Chuang, Chih-Pin Andrew; Argonne National Laboratory, United States

Consuelo, Garcea Serafina; University of Manchester, United Kingdom

Dobatkin, Sergey V.; IMET RAS, Russian Federation

Dudzinski, David; Derivation Research Laboratory Inc., Canada

Eftink, Benjamin P.; Los Alamos National Laboratory, United States
Egeonu, Uche A.; Xtiches Consult, Nigeria

Engelbrekt, Christian; Technical University of Denmark, Denmark

Farokhzadeh, Kora; United States

Fleuriault, Camille M.; Gopher Resource, United States

Gan, Bin; Northwestern Polytechnical University, China

Gao, Youping; Aerojet Rocketdyne, United States

Gelb, Jeff; Carl Zeiss X-Ray Microscopy Inc, United States

Goeken, Mathias; University Erlangen-Nuernberg, Germany

Guillet, Francois; French Alternative Energies and Atomic Energy Commission-CEA, France

Harris, William Carl; Zeiss X-ray Microscopy Inc, United States

Haskins, Adam W.; Metals Engineering and Testing Laboratory, United States

Hata, Satoshi; Kyushu University, Japan

Hayashi, Yujiro; Toyota Central Research \& Development Laboratories Inc., Japan

Hefferan, Christopher M.; RJ Lee Group, United States

Herzing, Andrew A.; National Institute of Standards \& Technology, United States

Hinshaw, Andrew L.; Arconic, United States

Hirayama, Kyosuke; Kyushu University, Japan

Holt, Nancy J.; Hydro Aluminium AS, Norway

Hosokawa, Akihide; McMaster University, Japan

Hybinette, Carl; Hybinette and Associates, United States

Jahazi, Mohammad; Ecole De Technologie Superieure, Canada
Jakobsen, Anders; Technical University of Denmark Physics, Denmark

Jang, Changheui; Korea Advanced Institute of Science \& Technology, South Korea

Jeffers, Elizabeth; Wyman-Gordon, United States

Joshi, Shailesh; Toyota, United States

Juul, Nicolai Y.; Technical University of Denmark, Denmark

Kakehi, Koji; Tokyo Metropolitan University, Japan

Ke, Liqin; Ames Laboratory, United States

Kemp, Craig; Eli Lilly \& Company, United States

Kim, Joon-il; Northwestern University, United States

Kim, Andrea; United States

Kimura, Hidehiko; Toyota Central Research \& Development Laboratories Inc., Japan

Kuwazuru, Osamu; University of Fukui, Japan

Landreau, Anthony; France

Langer, Stephen; National Institute of Standards and Technology, United States

Le Guilloux, Yann; Safran Paris-Saclay, France

Lee, Yueh-Lin; United States

Lee, Sang Hoon; FEl Company, United States

Li, Bo; Case Western Reserve University, United States

Lian, Junhe; RWTH Aachen University, Germany

Liu, Fengchao; Brigham Young University, United States

Liu, Zhilin; Central South University, China

Lobner, Dustin; Construction Forms, United States 
Loos, Peter John; Rice University, United States

Loughnane, Gregory T.; Wright State University, United States

Madi, Yazid; Mines ParisTech, France

Maire, Eric; University De Lyon, France

Margiotta, Jesse; Strategic Analysis, United States

McDonald, Samuel; University of Manchester, United Kingdom

McQuaid, Natasha; Roar Engineering, Canada

Mishra, Sushil; Indian Institute of Technology Bombay, India

Narayanan, Kris; Shell, United States

Nassiri, Ali; Ohio State University, United States

Nguyen, Thanh Tung; National Centre for Scientific Research, France

Nie, Ganggang; China

Pagan, Darren; Lawrence Livermore National Laboratory, United States

Palo, Gary; United States

Paranjape, Harshad; Colorado School of Mines, United States

Patterson, James E.; Brigham Young University, United States

Patterson, Brian M.; Los Alamos National Laboratory, United States

Pierce, Dean; Oak Ridge National Laboratory, United States

Pillay, Beverley A.; South32, South Africa

Povoden-Karadeniz, Erwin; Technische Universität Wien CDLESOP, Austria

Proudhon, Henry; Mines Paristech Centre Des Materiaux, France

Qian, Xiaofeng; Texas A\&M University, United States

Quey, Romain; Mines Saint-Etienne/ CNRS, France

Rockett, Angus A.; Colorado School of Mines, United States

Sahu, Sulata K.; Massachusetts Institute of Technology, United States
Sanchez-Segado, Sergio; University of Leeds, United Kingdom

Santerre, Renaud; Rio Tinto Alcan, Canada

Sarosi, Peter M.; ExxonMobil, United States

Sasikumar, Kiran; United States

Schreiber, Daniel K.; Pacific Northwest National Laboratory, United States

Schuurman, Sophie; Vishay Resistors Belgium, Belgium

Serrano, Itziar; Polytech Lille, France

Sharma, Hemant; Argonne National Laboratory, United States

Shiflet, Gary J.; University of Virginia, United States

Silwal, Bishal; Georgia Southern University, United States

Simons,Hugh; Technical University of Denmark, Denmark

Slater, Thomas; University of Manchester, United Kingdom

Smith, Franc B.; Bodycote, United States

Song, Jenn-Ming; National Chung Hsing University, Taiwan

Stewart, Derek; Western Digital, United States

Stringer, Craig; Atlas Pressed Metals, United States

Takeuchi, Akihisa; Japan Synchrotron Radiation Research Institute, Japan

Tan, Keqin; Australia

Taniyama, Akira; Nippon Steel \& Sumitomo Metal Corporation, Japan

Tari, Vahid; Carnegie Mellon University, United States

Tempel, Gina; University of Nevada Reno, United States

Tewari, Asim; Indian Institute of Technology Bombay, India

Thiagarajan, A.; Sri Manakula Vinayagar Engineering College, India

Thuo, Martin; lowa State University, United States

Tidrow, Steven; Alfred University, United States
Titarenko, Valeriy; University of Manchester, United Kingdom

Toohey, Caitlin M.; Hyster-Yale Group Inc., United States

Uchic, Michael; United States

Umada, Takateru; Chulalongkorn University, Thailand

Van Veen, Gerard; FEI Company, Netherlands

Villanova, Julie; European Synchrotron Radiation Facility, France

Villarreal, Julian E.; United States

Wan Ali, Wan Fahmin Faiz Bin; Universiti Teknologi Malaysia, Malaysia

Wang, Haibin; China

Wielewski, Euan; University of Glasgow, United Kingdom

Winiarski, Bartlomiej; University of Manchester, United Kingdom

Woracek, Robin; European Spallation Source, Sweden

Yakubovsky, Oleg; ArcelorMittal East Chicago, United States

Yang, Xiaogang; Argonne National Laboratory, United States

Yao, Zhihao; University of Columbia New York, United States

Yoshioka, Satoru; Kyushu University, Japan

Zander, Daniela; RWTH Aachen, Germany

Zhang, Yubin; Technical University of Denmark, Denmark

Zhang, Ning; United States

Zhao, Kejie; Purdue University, United States

Zhu, Hong; Arcelormittal Global Research \& Development, United States

Ziehmer, Markus; Helmholtz-Zentrum Geesthacht, Germany 